Rev. SINAPSIS, Edición № 14, Vol. 1, Julio 2019

\title{
Proyecto Educativo GENIALES TIC, con orientación a las competencias blandas
}

GENIALES TIC Educational Project, oriented to soft skills

\section{GENIALES TIC}

Judith Araújo de Paniza(1)

Universidad de la Sabana, Chía, Colombia,

Maestría en Proyectos Educativos mediados por TIC, juditharpa@unisabana.edu.co

Contacto: juditharpa@unisabana.edu.co

\section{Receptado 01/05/2019 Aceptado: 02/08/2019}

\section{Resumen}

Este artículo describe los resultados de una investigación evaluativa mixta con énfasis en lo cualitativo, que tiene como objetivo general de investigación, analizar los efectos del Proyecto Educativo mediado por TIC, GENIALES-TIC, en el desarrollo de competencias blandas de directivos docentes y docentes de la Institución Educativa Técnica Agropecuaria de Sincerín (INETASIN). Se analizan los efectos teniendo como base el modelo de Guskey, en relación con los objetivos específicos del proyecto. Tiene en cuenta la reacción de los participantes ante el Proyecto, los aprendizajes respecto al reconocimiento de talentos e inteligencias múltiples, el fortalecimiento del carácter y de la Inteligencia Emocional y la definición de sus proyectos de vida. También, se evalúan los efectos en la Institución Educativa, en el clima laboral, los cambios realizados en su Proyecto Educativo Institucional (PEI), en la aplicación de los nuevos aprendizajes en el aula de clases y en el aprendizaje y motivación de los estudiantes. El Proyecto fue evaluado como excelente por los directivos docentes y docentes con una calificación promedio de 4.86/5.00 y comentarios muy positivos que indican gran valoración del Programa. Los principales resultados evidencian aprendizajes, nuevas actitudes y comportamientos relacionados con las competencias trabajadas. Reportan mejoras en el clima institucional; han realizado ajustes al Proyecto Educativo Institucional PEI, para incorporar las competencias blandas intrapersonales e interpersonales. Manifiestan la intención de focalizar más la atención en los talentos de los estudiantes y agregar una asignatura para trabajar las competencias blandas desde preescolar hasta secundaria.

Palabras claves: Competencias, desarrollo humano, habilidades, Tecnología de la información y las comunicaciones- (TIC) 
Rev. SINAPSIS, Edición №14, Vol. 1, Julio 2019

ISSN $1390-9770$

\section{Abstract}

Educational Project GENIALES TIC, related with the development of soft skills

\section{GENIALES TIC}

This article is about the results of a mixed evaluative research with an emphasis on the qualitative paradigm, whose general objective is to analyze the effects of the Educational Project mediated by Information and Communication Technologies (ICT), GENIALES-TIC, in the development of soft skills of teachers and teaching directors of the Institution Educative Agricultural Technician of Sincerín (Institución Educativa Técnica Agropecuaria de Sincerín, INETASIN). The effects are analyzed based on the Guskey model, in relation to the specific objectives of the project. It takes into consideration the reaction of the participants to the Program itself, the learnings about multiple intelligences and the discovery of their main talents, as well as the strengthening of character, emotional intelligence and defining their personal life projects. The effects on the Educational Institution are also analyzed, as well as on the work environment, the changes on the Institutional Educational Program (PEI), the application of the new learnings in the classroom and the motivation of the students. The Program received an excellent review from directors and teachers, with a grade of 4.86/5.00, which indicates great value of the Program. As far as the learnings, it can be highlighted that they show more knowledge related to the skills they have worked on. They report improvements in the work climate; they have made important adjustments to the Institutional Educational Project PEI, to incorporate the competences of the Project. They express the intention to focus more attention on the talents of students and add a subject to work soft skills from preschool to High School.

\section{Keywords:}

Competencies, personal development, skills, Information and Communication Technologies (ICT)

\section{Introducción}

Las Instituciones Educativas suelen concentrar sus esfuerzos en el proceso de enseñanza aprendizaje de las asignaturas, lo que algunos autores llaman competencias duras, olvidando un poco las llamadas 
competencias blandas, que están relacionadas con el desarrollo del ser, sus habilidades personales y sociales, (Robinson, Aronica, 2015). Estos autores consideran que es necesario que el proceso de enseñanza aprendizaje sea más personalizado y esté más enfocado en los talentos naturales e intereses de los estudiantes.

En un estudio realizado en una escuela en Israel, en la que se trabaja la Inteligencia Emocional (IE) de los docentes, se demuestra que trabajar la IE y otras competencias blandas con los docentes, incide positivamente en ellos como personas y en su labor pedagógica, contribuyendo en el bienestar y desarrollo de los estudiantes (Dolev, Leshem, 2016).

En su libro Inteligencia Emocional, Goleman, (2013) nos relata algunas experiencias en colegios en los cuales se ha trabajado intencionalmente la Inteligencia Emocional y al comparar el comportamiento de los estudiantes con los de otras escuelas que no la han trabajado, se observa resultados muy positivos como, por ejemplo: que asumen más sus responsabilidades, se muestran más seguros de sí mismos, populares, sociales, colaboradores, comprensivos, considerados, democráticos. Tienen mejores habilidades para la resolución de conflictos, mejores resultados en las pruebas de desempeño, más tolerancia ante la frustración. Se evidencia también un clima de aula más positivo y menos problemas de drogadicción y delincuencia.

Se desarrolla un Proyecto Educativo mediado por TIC, GENIALES TIC con un enfoque de desarrollo humano centrado en la manera de ser, pensar, sentir y actuar de las personas. Se enfatiza en las competencias intrapersonales e interpersonales relacionadas con el conocimiento de sí mismos, de los talentos e inteligencias múltiples, el fortalecimiento del carácter e Inteligencia Emocional y en los Proyectos de vida. Se trabaja con los directivos y docentes para que se produzca un efecto cascada y ellos, a su vez, trabajen estas competencias con los estudiantes y padres de familia, tomando en cuenta las problemáticas del contexto, sus intereses y motivaciones

La UNESCO (2013), afirma que se requiere en Colombia de programas intencionalmente dirigidos hacia la formación del ser humano, que aborde el desarrollo integral de las personas en los 4 pilares del aprender: aprender a conocer, aprender a hacer, aprender a convivir y también, centrar de forma integradora, las bases para la formación del aprender a ser de las personas. 
El Proyecto Educativo GENIALES TIC propone fortalecer los indicadores de calidad educativa, con un modelo de desarrollo integral de las competencias blandas, que potencie al ser humano hacia la búsqueda de la excelencia y la felicidad enfatizando en el saber ser y saber convivir. (competencias intrapersonales e interpersonales).

Al respecto, Papalia, Wendkos, Duskin, (2010) expresan:

Los estudios de los científicos del desarrollo afirman que son tres los ámbitos principales del desarrollo: físico, cognoscitivo y psicosocial. El crecimiento y desarrollo del cuerpo y el cerebro, las capacidades sensoriales y motrices y la salud son parte del desarrollo físico. El aprendizaje, la atención, la memoria, el lenguaje, el pensamiento, el razonamiento y la creatividad forman parte del ámbito cognoscitivo y las emociones y la personalidad hacen parte del ámbito psicosocial. Son ámbitos interrelacionados, el desarrollo de cada uno, afecta el de los demás. (p.5)

En la implementación se inicia el proceso con los directivos docentes y docentes y luego se analiza la forma de transferir esas competencias al salón de clases y al aprendizaje de los estudiantes. Se busca contribuir a mejorar el problema educativo e incidir en el cambio del modelo pedagógico, para que se concentren en la formación de la persona de manera integral, dando como resultado que los miembros de la comunidad educativa estén en capacidad de formular proyectos de vida alcanzables y prósperos, en medio de un clima educativo favorable y contribuyendo al desarrollo de sus propios talentos y fortalecimiento del carácter y de la inteligencia emocional. En este sentido, afirma Araújo (2017):

Los talentos naturales y la misión de vida no se inventan, se descubren. Quien logra descubrirlos, desarrollarlos y hacerlos sus herramientas para ganarse la vida, se concentra y dirige sus energías con disciplina y tiene la oportunidad de convertirse en un fuera de serie, en un genio. ( p. 30)

Para Robinson \& Aronica (2016), trabajar estas competencias, es un aporte a los procesos educativos donde se valora que "la finalidad de la educación es capacitar a los alumnos a que comprendan el mundo que les rodea y conozcan sus talentos naturales con objeto de que puedan realizarse como individuos y convertirse en ciudadanos activos y compasivos" (p.24).

A nivel nacional, la Organización para la Cooperación y el Desarrollo Económico OCDE (2016), expresa que para mejorar la calidad y la pertinencia de los resultados del aprendizaje, Colombia "primero que 
todo, necesita fijar expectativas claras de los valores, los conocimientos y las competencias que deben adquirir los estudiantes en cada etapa del ciclo educativo" (OECD, 2016, p. 16).

El reto departamental en materia de calidad educativa es mayúsculo, máxime que el Índice Sintético de Calidad para Bolívar (ISC) en 2016, lo ubicó en el tercer puntaje más bajo del país. La situación demanda una intervención integrada con la detección de los talentos, que inspire el desarrollo de nuevas generaciones, con más capacidades y competencias para adaptarse a las demandas de una región en desarrollo.

El Proyecto incluye la construcción de una página interactiva, Plataforma LMS, de desarrollo humano ajustada a las necesidades personales de los participantes: aulavirtual.fundaciongeniales.org para hacer un acompañamiento al Proyecto a través de las TIC. Las TIC contribuyen con el aprendizaje personalizado, desarrollo de autonomía, el aprendizaje colaborativo y la dinamización del aprendizaje con las ayudas multimedia, permitiendo la accesibilidad al conocimiento, sin limitaciones por las distancias y el tiempo. "Estamos en una situación innegable para utilizar muchos recursos creativos y tecnológicos con el fin de cambiar la situación actual” (Robinson \& Aronica, 2016, p.24).

El objetivo general de la investigación evaluativa del Proyecto es: Analizar los efectos del Proyecto GENIALES TIC en el desarrollo de competencias blandas de directivos docentes y docentes de la Institución Educativa Técnica Agropecuaria de Sincerín (INETASIN).

Los objetivos específicos integrados con el modelo Guskey son: identificar los niveles de satisfacción que tiene el programa en los directivos docentes y docentes; reconocer los aprendizajes en relación al autoconocimiento de sus talentos e inteligencias múltiples, el fortalecimientos del carácter y de la Inteligencia Emocional y la definición de sus proyectos de vida; reconocer los cambios en la Institución Educativa; identificar cómo inciden en la práctica pedagógica y evidenciar el aprendizaje transferido a los estudiantes en los temas trabajado en el Proyecto GENIALES TIC.

\section{Materiales y métodos:}

Se realiza una investigación mixta (incluye instrumentos cuantitativos y cualitativos) con énfasis cualitativo. El diseño metodológico es de tipo evaluativo aplicando los cinco niveles del modelo de 
Guskey, con el objetivo de analizar los efectos del Proyecto Educativo mediado por TIC, GENIALESTIC en el desarrollo de competencias blandas en los directivos y docentes de la Institución Educativa INETASÍN.

En la Tabla 1 se consolida la información respecto al objetivo de cada uno de los niveles de Guskey, combinando con los objetivos del proyecto y con los indicadores establecidos para medir los resultados por cada objetivo; se establece la pregunta de investigación para cada nivel. También, se incluyen las técnicas, la fecha de aplicación y número de personas que participan en cada instrumento. 
Tabla 1. Materiales y métodos evaluación Proyecto Educativo con modelo Guskey

\begin{tabular}{|c|c|c|c|c|c|c|c|c|c|c|c|}
\hline \multirow{2}{*}{\multicolumn{2}{|c|}{$\begin{array}{l}\text { PKLC HUMIFN IG] } \\
\text { FORMULADO PARAEL } \\
\text { MODELO }\end{array}$}} & \multicolumn{4}{|c|}{ DIAGNÓSIICO } & \multicolumn{4}{|c|}{ IMPLEMENTACIÓN } & \multirow{2}{*}{\multicolumn{2}{|c|}{$\begin{array}{c}\text { POSTERICR } \\
\text { Nivel5. Aprendizaje delos } \\
\text { estudiantes }\end{array}$}} \\
\hline & & \multicolumn{3}{|c|}{$\begin{array}{l}\text { Nivel 1. La rosción de los } \\
\text { porticipontes }\end{array}$} & $\begin{array}{c}\text { Mivel 2. E } \\
\text { a prendizajede } \\
\text { los participantites. }\end{array}$ & \multicolumn{2}{|c|}{$\begin{array}{l}\text { Nivd 3. El soperte y } \\
\text { cambio orranizadional }\end{array}$} & \multicolumn{2}{|c|}{$\begin{array}{l}\text { Nived 4. Uso de nueves } \\
\text { conocimientos y habilidader }\end{array}$} & & \\
\hline \multirow{2}{*}{\multicolumn{2}{|c|}{$\begin{array}{l}\text { PREGUNTASE } \\
\text { INUK_ALXJRS }\end{array}$}} & PRFIINTA & OBETIVO & PRFCLINTA & OBIETINO & PRFCAINTA & ORIFTMO & PRFGIINTA & ORIFTMD & PRFININA & ORIFIMV \\
\hline & & 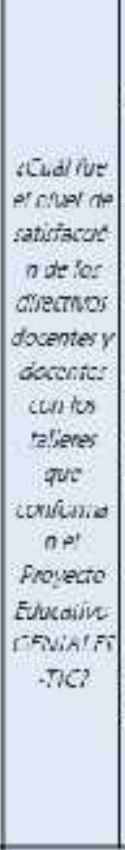 & 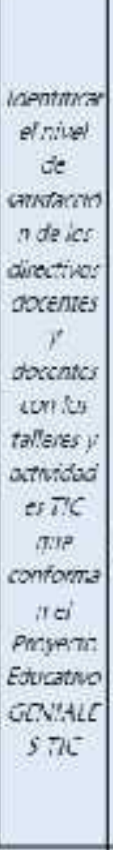 & 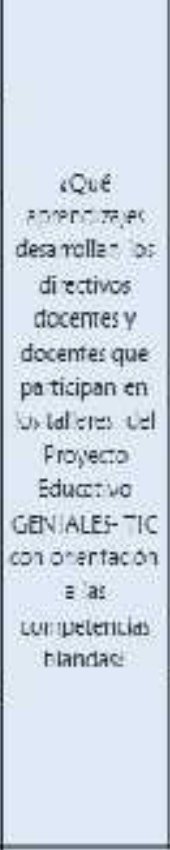 & 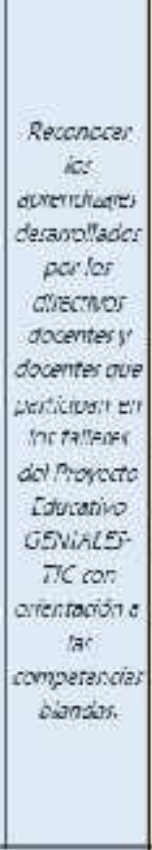 & 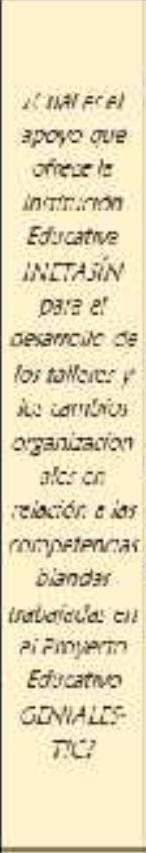 & 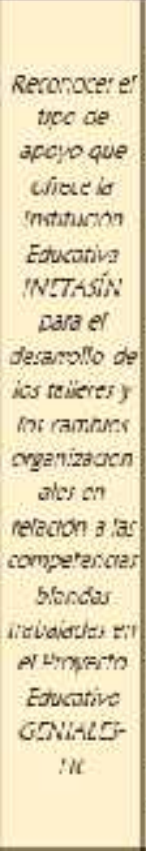 & 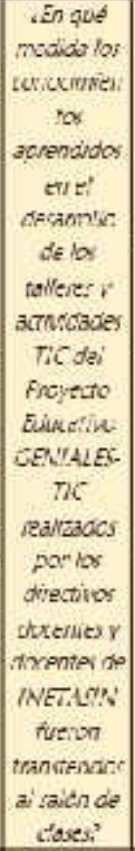 & 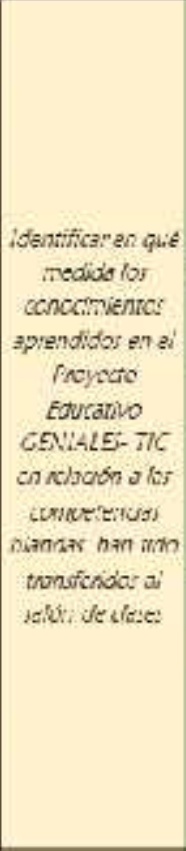 & 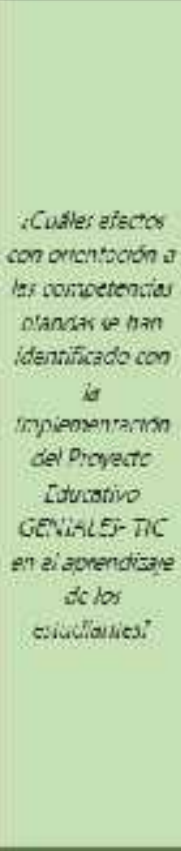 & 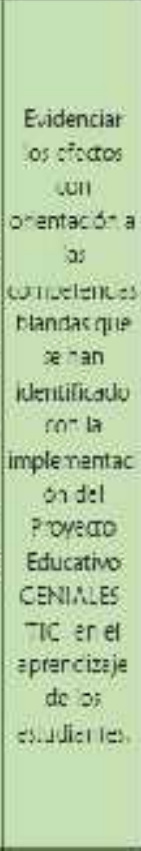 \\
\hline 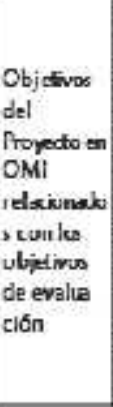 & 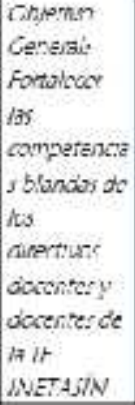 & & & \multicolumn{2}{|c|}{ 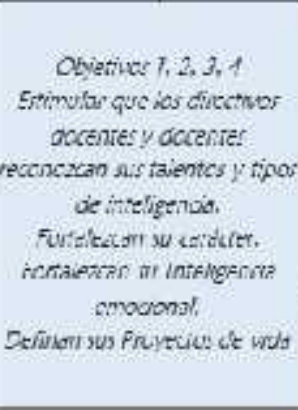 } & \multicolumn{2}{|c|}{ 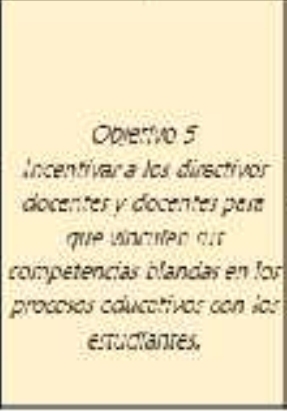 } & \multicolumn{2}{|c|}{ 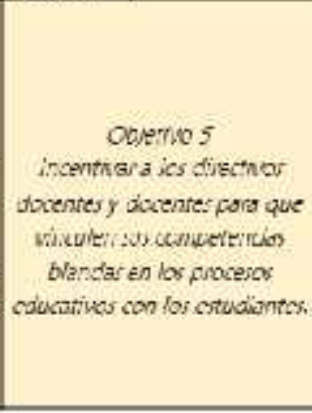 } & 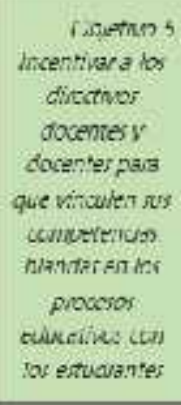 & \\
\hline
\end{tabular}




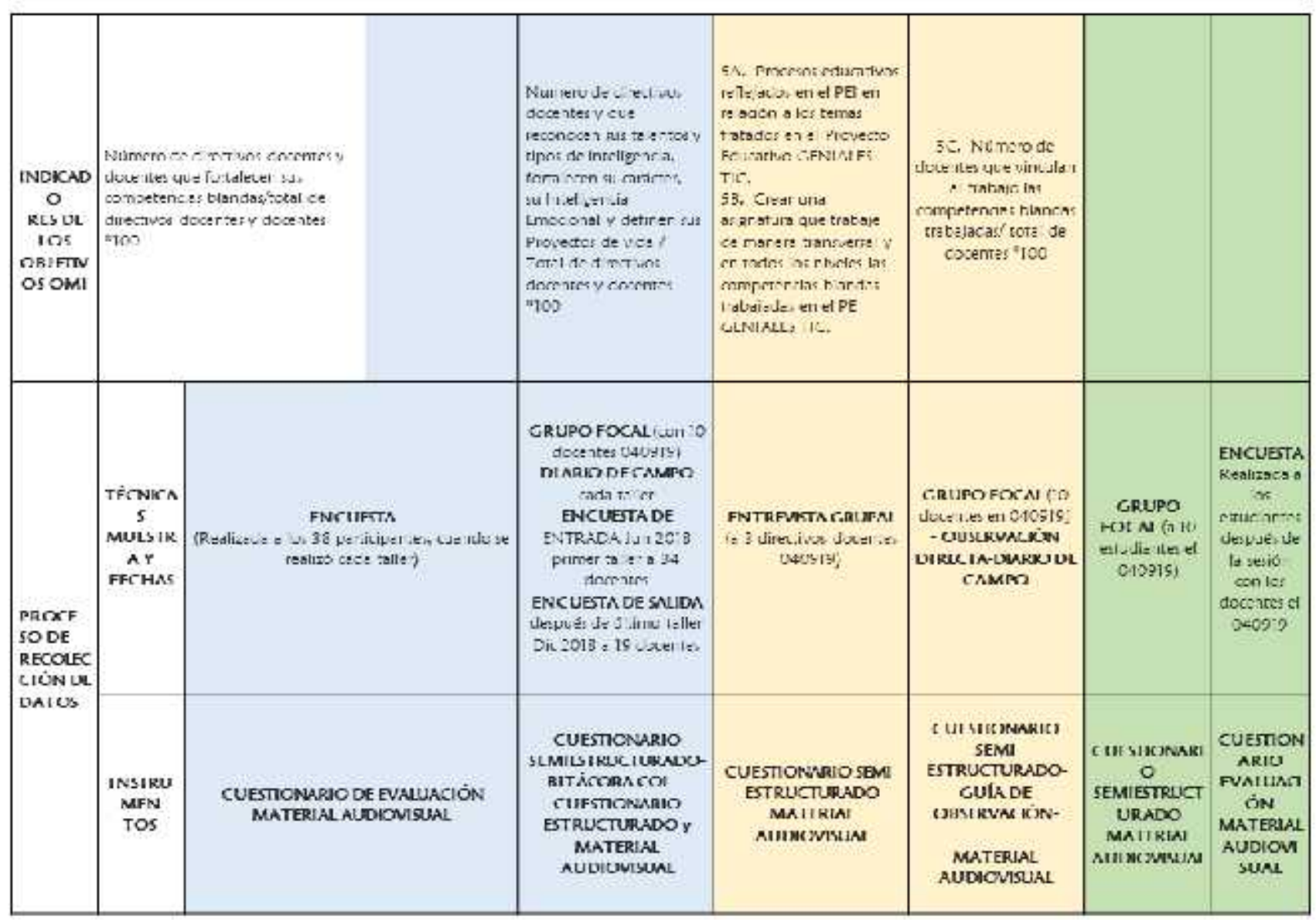

\section{Población y muestra:}

La investigación se realiza en la Institución Educativa Técnica y Agropecuaria de Sincerín INETASÍN, participando la mayoría de los directivos (3) y docentes (35). Sincerín es un corregimiento del municipio de Arjona, localizado en el Departamento de Bolívar(Colombia). Con ellos se implementa el programa y luego se evalúan los efectos.

\section{Resultados}

La evaluación se realiza con base al modelo de Guskey, triangulando la información con los objetivos del proyecto. Cada nivel tiene sus propias técnicas e instrumentos, pero también se cruza la información de acuerdo con las respuestas y con el programa virtual MAXQDA se va realizando la segmentación de la información. 


\section{Nivel 1. Reacción de los participantes}

\section{Pregunta de evaluación: ¿Cuál fue el nivel de satisfacción de los directivos docentes y docentes con los talleres que conforman el Proyecto Educativo GENIALES-TIC?}

El instrumento aplicado para este nivel es un cuestionario estructurado mixto con preguntas cuantitativas y cualitativas. Se mide el contenido del programa, la aplicabilidad a la vida diaria, conocimiento y actitud participativa del facilitador, la comunicación y comportamiento del grupo, la amenidad de las sesiones, la calidad y cantidad del material impreso, las instalaciones y ayudas audiovisuales.

En el balance del promedio general de la evaluación, la escala ubica un $78 \%$ en el nivel excelente, un $17 \%$ en nivel muy bueno y un $5 \%$ en el nivel bueno. Se destaca que en los niveles regular y deficiente no se registraron respuestas. Es decir, el 95\% de los participantes se ubican en el máximo nivel de las escalas, evidenciando en alto grado de favorabilidad y cumplimiento de expectativas con su participación en el Programa Geniales. El promedio de evaluación general del programa corresponde a un 4.86/5.00.

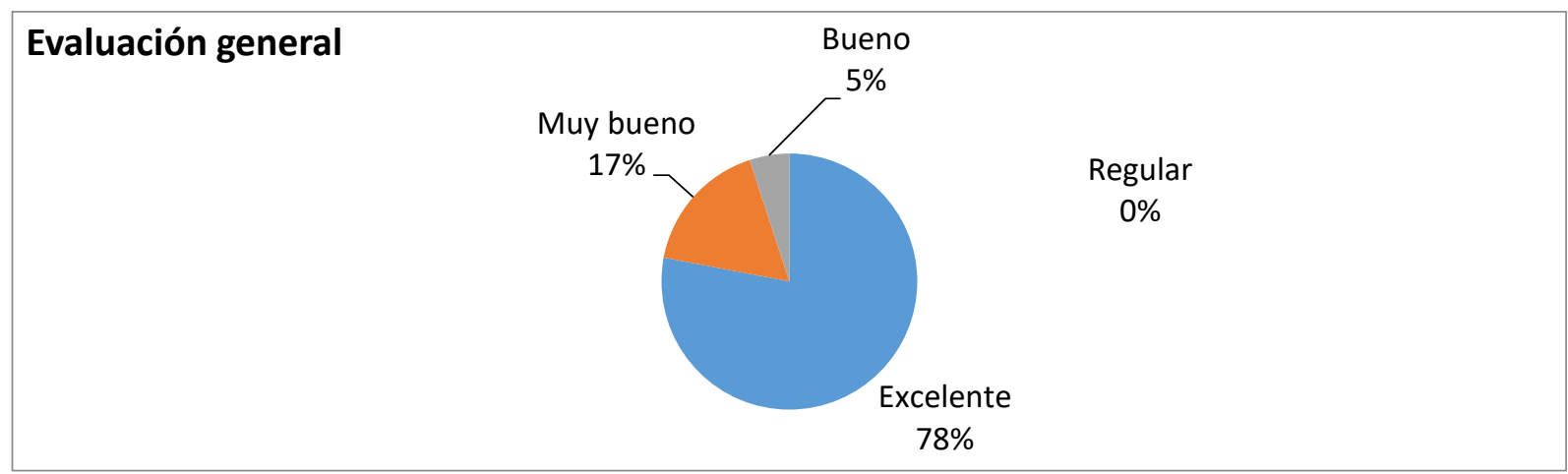

Figura 1. Promedio general de la evaluación.

\section{Análisis cualitativo:}

Para el análisis cualitativo se toma en cuenta los comentarios escritos en la evaluación y comentarios expresados con los otros instrumentos relativos a la reacción de los participantes frente al Proyecto.

Algunos de los comentarios referentes a la valoración del temario son:

- "Ayudan a cambiar al ser humano, a transformarlo y a mejorar este mundo." 
Rev. SINAPSIS, Edición № 14, Vol. 1, Julio 2019

- "Excelente, motivadora y de ayuda personal en las emociones que manejamos en nuestra vida". "Excelente capacitación; por favor seguir ampliando más en estas temáticas que ayudan a nuestro crecimiento personal y profesional". "Buen contenido que ayuda a rediseñar maestros geniales".

- "Todos queremos felicitarlas, este es el único proyecto que se le ha visto que surte todos sus procesos. En mi parte personal y profesional todos los temas que se desarrollaron aquí han sido útiles para mí, me sirvió mucho el de la inteligencia emocional, cómo fortalecer mi carácter, cómo manejar las situaciones en los salones de clase porque a veces con los estudiantes actuamos una manera que no es la correcta y a través de todos estos módulos he venido mejorando mis prácticas pedagógicas”.

Nivel 2. El aprendizaje de los participantes

Pregunta de evaluación: ¿Qué aprendizajes desarrollan los directivos docentes y docentes que participan en los talleres del Proyecto Educativo GENIALES- TIC con orientación a las competencias blandas?

Análisis cuantitativo -encuestas de Conductas de entrada y salida:

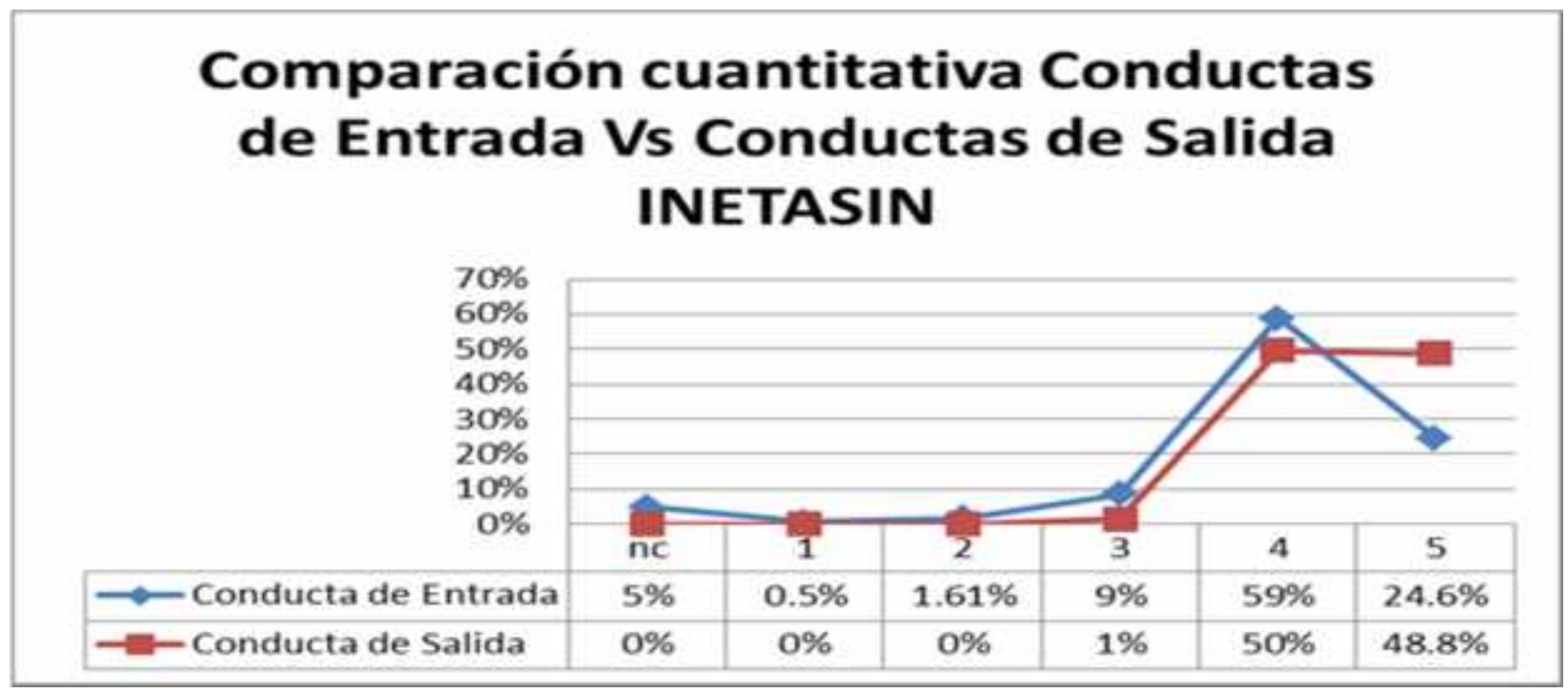

Figura 2. Comparación respuestas conductas de entrada y salida. 
En la figura 2 se puede inferir el análisis cuantitativo, en el que se muestra un desplazamiento en las respuestas a los cuestionarios de conducta salida hacia números superiores en relación con los registrados en los que medían la conducta de entrada, es decir que señalan mayores conocimientos respecto a los temas tratados en el Programa. Los resultados se evidencian también en el análisis cualitativo con base a las preguntas abiertas.

Se triangulan los resultados obtenidos a través de los diferentes instrumentos de evaluación para analizar los aprendizajes en relación con los objetivos del Proyecto Geniales TIC:

\section{Objetivo 1. Estimular que los directivos docentes y docentes reconozcan sus talentos y tipos de inteligencia:}

Los directivos docentes y docentes manifiestan a través de los diferentes instrumentos más conocimientos de sí mismos, de sus talentos e inteligencias múltiples. Algunas de sus afirmaciones fueron:

$\checkmark$ "Este Programa me ayudó a descubrir talentos en lo personal y en los estudiantes lo que contribuyó a mejorar su desempeño escolar. Comprendí que es importante conocerse a sí mismo y conocer mejor los talentos de los estudiantes".

$\checkmark$ “Aprendí a darle importancia a cada uno de mis talentos, trabajarlos con esmero y utilizarlos como fortalezas para mejorar en mi vida personal y profesional que me permita crecer y brillar con luz propia. Además, la importancia de reconocer las diferentes inteligencias presentes en cada individuo y que como educadora debo procurar que cada niño reconozca sus talentos y trabaje en el verdadero desarrollo de éstos".

$\checkmark$ "Mucha incidencia en el quehacer como docente pues los temas y actividades tratados sobre las inteligencias múltiples que tienen mucha aplicación a nivel personal y con los estudiantes. Videos motivadores, frases y reflexiones para conocer nuestras inteligencias y potencialidades”. 


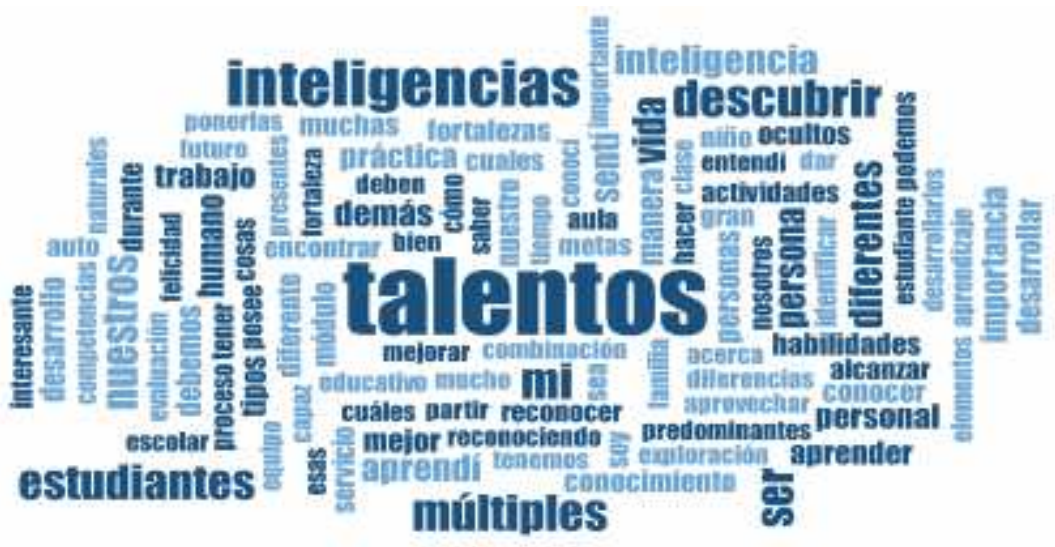

Figura 3. Nube de palabras sobre aprendizajes de talentos e inteligencias múltiples. Elaboración propia con programa MAXQDA.

\section{Objetivo 2: Fortalecer el carácter}

\section{Algunas expresiones sobre los aprendizajes respecto a este tema:}

$\checkmark$ "Vimos varios aspectos que nos ayudan a formar y a fortalecer nuestro carácter como son la motivación, voluntad, disciplina, alegría, que estos aspectos nos guían hacia lo que se debe y lo que no se debe en cada situación”.

$\checkmark \quad$ "Comprendí que, para alcanzar la felicidad plena, debo ser consciente del carácter que me define como una persona única y que para fortalecerlo debo tener en cuenta los principios, valores y virtudes."

$\checkmark \quad$ "Poniendo en práctica las 4 virtudes cardinales: Prudencia, Justicia, Fortaleza y Templanza, en las 4 dimensiones, Corporal, Espiritual, Mental, Emocional podré trabajar siempre mi felicidad y de los que me rodean".

$\checkmark$ "Qué para construir un carácter fuerte, es necesario tener en cuenta los principios, valores y virtudes, para que la razón tome el control de las situaciones actuando con sabiduría y alcanzar en la tierra el fin para el cual fuimos creados". 


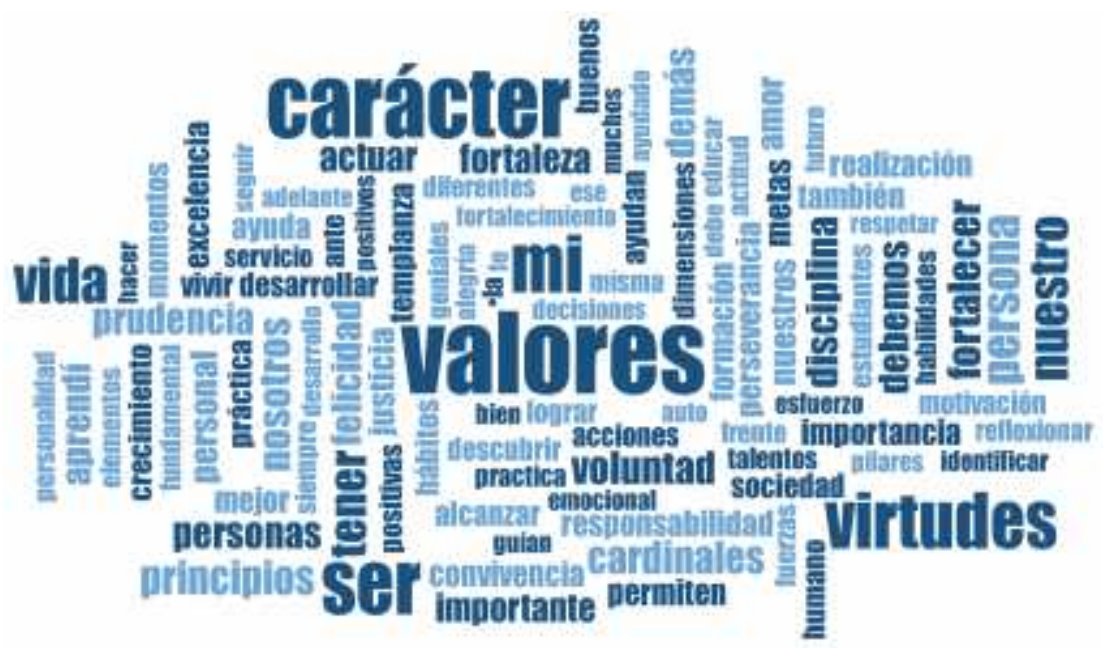

Figura 4. Nube de palabras fortalecimiento del carácter. Elaboración propia con programa MAXQDA.

\section{Valores que resaltan los docentes en sus aprendizajes:}

Confianza en sí mismo, fortaleza, pasión, alegría, curiosidad, satisfacción personal, tenacidad, esfuerzo, prudencia, cooperación, armonía, creatividad, innovación, conocimiento de sí mismo y a los compañeros de trabajo, perseverancia, servicio, disciplina, compromiso, fortaleza, perseverancia, autodisciplina, constancia, responsabilidad, equidad, carisma, respeto, la valoración del otro, aceptación de diferencias, organización, planeación, valentía para luchar por las metas, amor, motivación, esfuerzo, virtudes cardinales, entusiasmo, autocontrol, iniciativa, paciencia, tolerancia, liderazgo, solidaridad, serenidad, equilibrio, estabilidad, creatividad, proactividad, virtudes sobrenaturales, cardinales y humanas, pasión, respeto mutuo, esperanza.

\section{Objetivo 3 Fortalecer la Inteligencia Emocional}

\section{Algunas afirmaciones de los directivos docentes y docentes respecto a este tema:}

$\checkmark$ "Sentí satisfacción con mi desarrollo personal, no desconociendo que hay que seguir fortaleciendo algunos componentes que ayuden en el desarrollo de procesos en el aula; debemos brindar a nuestros muchachos seguridad, mensajes que los lleven a la reflexión y razonamiento sobre control y reconocimiento de sus emociones básicas que ayuden a la construcción de seres felices que propendan por una sana convivencia”. 
$\checkmark$ "Que en las aulas de clases los estudiantes necesitan docentes motivados, con buenas relaciones personales, ejemplo a seguir para ellos, agentes de cambio para transformar la educación, la inteligencia emocional hace parte de las personas exitosas."

$\checkmark$ "Aprendí sobre los diferentes aspectos que involucra la inteligencia emocional, entendida como la habilidad social que nos permite manejar nuestras emociones de manera exitosa."

$\checkmark$ "Esta inteligencia identifica los estados de ánimos propios y ajenos, las relaciones sinérgicas que implican la construcción de relaciones significativas en otras personas". También nos ayuda a desarrollar estas habilidades emocionales en los estudiantes para que tengan buenas relaciones".

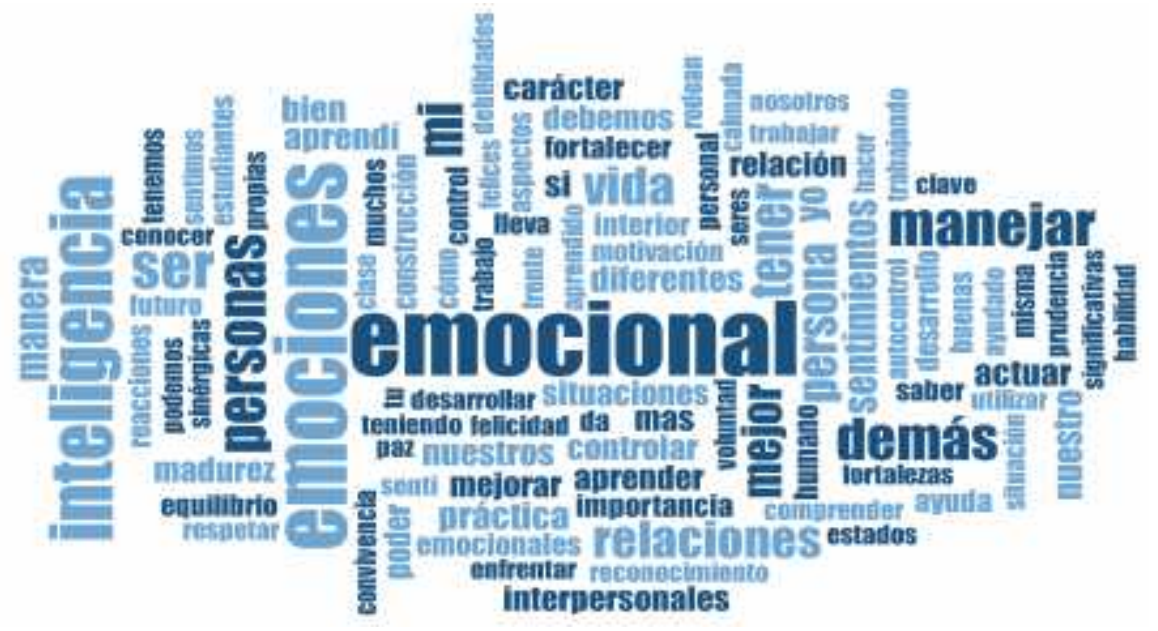

Figura 5. Nube de palabras aprendizajes Inteligencia emocional. Elaboración propia con programa MAXQDA.

\section{Objetivo 4 Definir sus Proyectos de vida}

$\checkmark$ "Me ayudó a saber quiénes somos y para dónde vamos, es importante si queremos conseguir nuestros sueños u objetivos; proyectamos significa plantear o trazar el camino por recorrer, a sabiendas que no será fácil pero tampoco imposible de lograr".

$\checkmark$ "Nuevamente me vi frente a un proceso de autoevaluación, que permitió focalizar prioridades en mi desarrollo personal e identificar aspectos a corregir para que mi carácter sea establecido y se logren realizar o ejecutar avances en mi proyecto de vida".

$\checkmark$ "Durante este módulo revisamos la importancia de construir proyectos de vida basados en principios y valores que orienten la consecución de nuestros objetivos a partir de la formulación de estrategias 
creativas. Lo anterior supone el involucramiento sinérgico de cada una de las áreas de nuestro ser (físico, mental, familiar, emocional, espiritual, profesional, entre otras). "Definí mi misión, visión, valores".

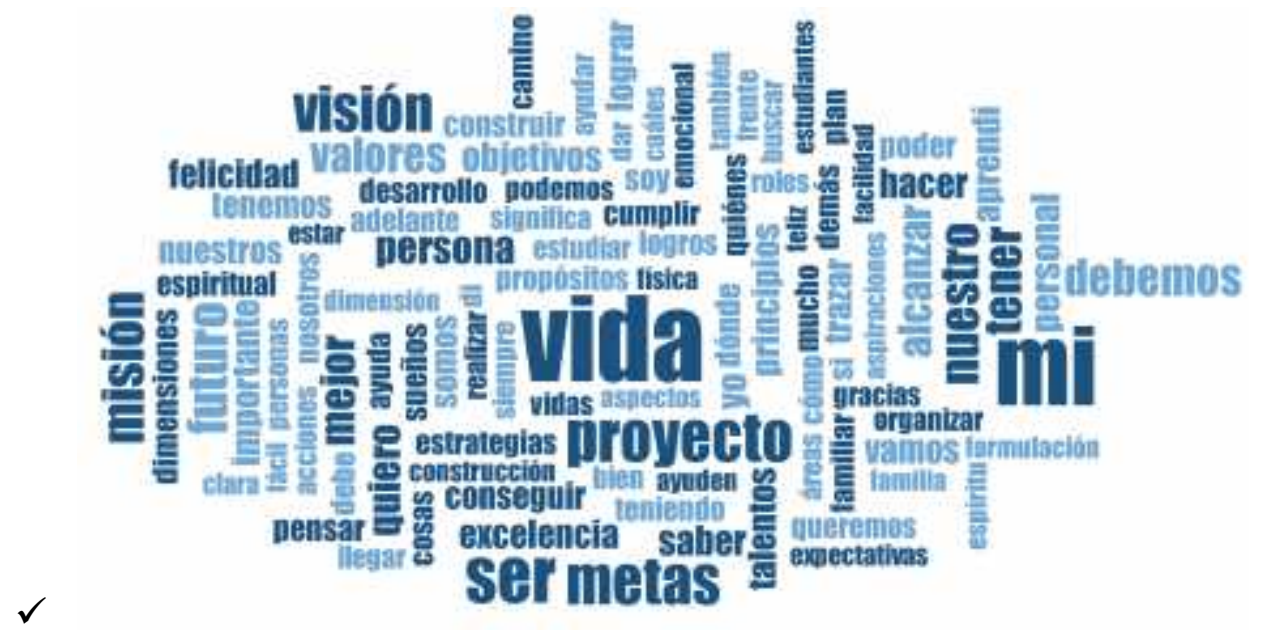

Figura 6. Nube de palabras en aprendizajes sobre Proyecto de vida. Elaboración propia con programa MAXQDA.

\section{Nivel 3. El soporte y cambio organizacional}

Pregunta de evaluación: ¿Cuál es el apoyo que ofrece la institución Educativa INETASÍN para el desarrollo de los talleres y los cambios organizacionales en relación a las competencias blandas trabajadas en el Proyecto Educativo GENIALES- TIC?

Los directivos docentes y docentes de la IE INETASÍN fueron muy colaboradores y estuvieron siempre prestos a contribuir con aspectos relacionados con la planeación y ejecución de los talleres y demás actividades del Proyecto GENIALES-TIC. Hemos también contado con el concurso de la Gobernación de Bolívar y su Secretaría de Educación quienes han participado activamente ofreciendo todo su apoyo.

Dentro del Proyecto, se trabaja con los directivos docentes el PEI de la Institución Educativa para analizar los cambios que van a realizar a la luz de los conceptos que se trabajaron en GENIALES TIC. Se reflejan cambios significativos en la misión, visión, valores y se considera la posibilidad para que los docentes incluyan de manera más intencional las competencias blandas y todos los temas del proyecto en el aula de clases. Este año se continúa con ese propósito para encontrar la forma que más se adecúe al pensum educativo e incorporar una asignatura que trabaje estos conceptos de manera transversal durante todo el período escolar. 
Rev. SINAPSIS, Edición № 14, Vol. 1, Julio 2019

ISSN $1390-9770$

Expresan algunos directivos docentes y docentes:

$\checkmark$ "Yo creo que hay una primera parte que tiene que ver con el trabajo realizado en el PEI que es una carta de navegación. Estamos hablando de una proyección del desarrollo que tiene en cuenta todos los elementos. Creo que todos los docentes, en medio del amor que tienen por el desarrollo de su trabajo, han tenido el compromiso de participar en todos los talleres, han demostrado su deseo de aprender, de apropiarse de los conocimientos para garantizar que la educación que están transmitiendo a sus estudiantes tenga el dinamismo, reconozca los talentos y todas esas herramientas que nos han ayudado a aprender; la semilla que están dejando en Sincerín, tendrá continuidad.”

$\checkmark$ "Aprendí a realizar procesos curriculares que ayuden a los estudiantes a potencializar sus inteligencias".

$\checkmark$ “Crear y ejecutar estrategias que favorezcan el desarrollo de talentos o inteligencias en nuestros estudiantes y evaluarlos de manera formativa, reconociendo sus diferencias y teniendo en cuenta sus estilos de aprendizaje".

\section{Nivel 4. Uso de los nuevos conocimientos y habilidades en el aula de clases}

Pregunta de evaluación: ¿En qué medida los conocimientos aprendidos en el desarrollo de los talleres y actividades TIC del Proyecto Educativo GENIALES- TIC realizados por los directivos docentes y docentes de INETASÍN fueron transferidos al salón de clases?

Los docentes han aprovechado sus nuevos conocimientos y habilidades en el aula de clases, aunque solo están iniciando el proceso y lo expresan en estas palabras:

$\checkmark$ "Bueno yo hablo de lo que está sucediendo con la primaria; hemos visto cambios porque hay varios docentes que aplican los aprendizajes que se han recibido de Geniales, se ha visto el cambio en los docentes, en su práctica pedagógica en los talentos. Bueno la verdad es que los valores, la forma en la que se hacen el trabajo sobre el quehacer diario de los docentes, su responsabilidad también ha cambiado son muy responsables, tienen mucho sentido de pertenencia”.

$\checkmark$ "Los efectos que a largo plazo se han venido reflejando en nuestro quehacer diario; en el grupo de docentes, por ejemplo, se creó el sentido de responsabilidad y en la aplicación de tema que tienen que ver con Geniales en el aula de clases. Precisamente hoy pudieron ver parte de ese proceso que los docentes estamos cambiando en la institución". 
$\checkmark$ "Dentro de los aportes, el descubrimiento de los talentos ahora está presente en nuestra evaluación estudiantil, estamos preguntando cuáles son los talentos, qué hay en la casa, mostrando los talentos de personajes que nosotros como institución no habíamos retomado en ese camino dentro del proceso educativo".

$\checkmark$ "Son muchas las herramientas que podemos ofrecerle a nuestros estudiantes que nos permitirán desarrollar competencias asociadas a sus talentos, convirtiéndonos en agentes transformadores del proceso educativo".

$\checkmark$ "Los cambios que se han visto en el comportamiento de los estudiantes, en los docentes, ahora son muy unidos, trabajan entre sí”.

$\checkmark \quad$ "A veces en el aula de clases, tenemos niños difíciles y lo impacientan a uno. Ahora estoy tratando acercarme un poco más a ellos. Ahora son más cercanos a mí, a veces los regaños lo que lograban era separarlos y que le cojan fastidio a uno, entonces estoy aplicando esta forma de tratarlos con mejores resultados.

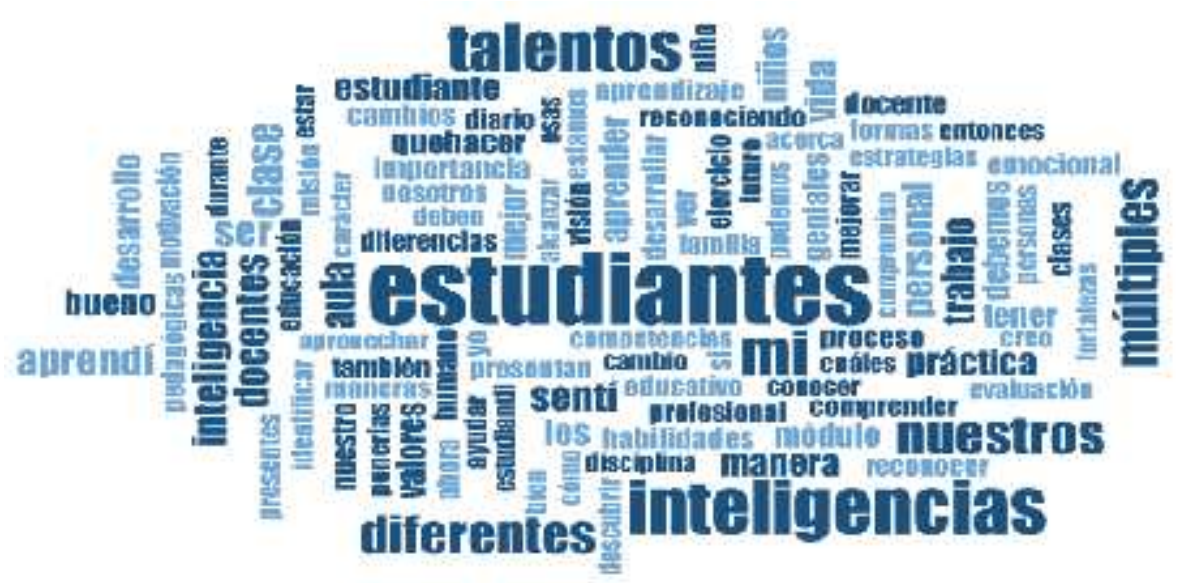

Figura 7. Nube de palabras sobre el uso de los nuevos conocimientos y habilidades en el aula de clases. Elaboración propia con programa MAXQDA

Nivel 5. Aprendizaje de los estudiantes

Pregunta de evaluación: ¿Cuáles efectos con orientación a las competencias blandas se han identificado con la implementación del Proyecto Educativo GENIALES- TIC en el aprendizaje de los estudiantes? 
$\checkmark$ Se pudo identificar importantes aprendizajes relacionados con la valoración de lo que son y el deseo de cumplir sus sueños y promesas, con aprender a compartir y llevarse bien con los demás. Le dan importancia al desarrollo de sus talentos, a tener mejores rendimientos académicos.

$\checkmark$ Afirma la directora: "Pude ver que los niños se sienten comprometidos, todos querían participar, están perdiendo el miedo y eso bueno. Se ve que sí están haciendo el trabajo y está dando los resultados con los niños".

$\checkmark$ Algunos niños ya plantean metas específicas: "Ganar los años sin perder una materia". "Yo quiero ser doctor, para salvar vidas". "Yo quiero ser futbolista, para llegar a un club". "Yo quiero ser abogada". "Yo quiero ser doctora para ayudar a los enfermos que están acostados en una cama". Yo quiero ser ginecóloga. "Yo quiero tener una Fundación en la que pueda ayudar a niños con síndrome de Down". "Yo quiero ayudar a los niños que no tienen un hogar que los han abandonado que no tienen familia que no tienen amor".

$\checkmark \quad$ "Que siempre hay que estudiar que debemos seguir adelante con nuestros estudios, llevábamos las notas bien abajo, estábamos perdiendo el interés por estudiar. Y Geniales nos enseñó, a volver a tener ganas de estudiar".

\section{Discusión}

Los resultados cuantitativos muestran un nivel alto de valoración del Proyecto Educativo GENIALES TIC por parte de directivos docentes y docentes de la IE INETASÍN. En el aspecto cualitativo también se puede evidenciar que los docentes están utilizando mejor las competencias blandas trabajadas en el proyecto en su quehacer cotidiano y han compartido con los estudiantes sus conocimientos, lo que se empieza a reflejar en cambios personales, en la Institución educativa, en su labor pedagógica y en los aprendizajes de los estudiantes, incluso en su ámbito familiar y social.

Respecto a los efectos en el aprendizaje de los participantes y los objetivos del Proyecto fueron muchos los aprendizajes y se vieron reflejados en conocimientos, actitudes, comportamientos, respecto a todos los objetivos del Proyecto.

Respecto a estimular el conocimiento de sí mismos, de sus talentos e inteligencias múltiples, se valida que los talentos naturales son lo que hace a cada persona única e irrepetible y que su mayor potencial está en concentrarse en ellos y generar fortalezas, dedicándoles tiempo y esfuerzo a su desarrollo. (Buckingham \& Clifton, 2011); también lo que afirma Robinson (2016) "Encontrar nuestro Elemento 
(término muy relacionado con los talentos) es fundamental para nosotros como individuos y para el bienestar de nuestra comunidad". (p.298)

Con respecto al fortalecimiento del carácter y de la Inteligencia emocional expresa Navarro, (2016) que el dominio propio se refiere a la autodisciplina, autocontrol y al manejo adecuado a los sentimientos; Goleman (2015) concluye, haciendo alusión a un teórico de la Universidad de Washington, Amitai Etzioni, que las escuelas tienen un papel muy importante en la formación del carácter contribuyendo con la autodisciplina y la empatía, lo que genera también compromisos cívicos y morales.

Con respecto a la construcción de un Proyecto de vida, Covey,(2010) expresa: "Cuan distintas son nuestras vidas cuando sabemos qué es lo verdaderamente importante para nosotros, y, manteniendo ese cuadro en mente, actuamos cada día para ser y hacer lo que en realidad nos interesa"( p.124).

En lo referente al soporte organizacional y a los cambios dentro de la Institución, cabe resaltar que la Institución Educativa fue muy participativa, todos los directivos docentes y docentes se involucran en el Proyecto, los directivos docentes participan activamente en las sesiones de resignificación del Proyecto Educativo Institucional PEI, se hacen ajustes, se mide y se reconocen avances en el clima organizacional y están aplicando los conceptos, estrategias, herramientas, hábitos trabajados en el Proyecto Geniales TIC. Se programa instituir una asignatura que trabaje de manera transversal los conocimientos aprendidos en el Proyecto durante todo el año escolar en los distintos niveles académicos y también para los padres de familia.

En relación al uso de los nuevos conocimientos y habilidades; se inicia la aplicación de los temas trabajados en GENIALES TIC en el salón de clases con sus estudiantes; se tiene planeado dejarlo institucionalizado de manera permanente, por los efectos positivos que tienen tanto en los profesores como en sus estudiantes, mejorando incluso las relaciones y el clima entre ellos.

Analizando los efectos en el aprendizaje de los estudiantes, respecto a las competencias blandas trabajadas con los directivos docentes y docentes de INETASÍN, las evidencias que se observaron tanto en niños de primaria como de bachillerato, es que ha sido muy positiva la aceptación y la asimilación de los conceptos, las actitudes y los comportamientos relativos al Proyecto Geniales TIC.

Es importante para los efectos en el largo plazo, que se siga un esfuerzo consistente aplicando los conocimientos en el quehacer cotidiano de la institución. Se ratifica lo encontrado también por Dolev, 
Rev. SINAPSIS, Edición № 14, Vol. 1, Julio 2019

Leshem, (2016) respecto a la incidencia positiva de trabajar la Inteligencia emocional y demás competencias blandas en los docentes incidiendo positivamente en ellos como personas y en su labor pedagógica y contribuyendo también en el bienestar y desarrollo de los estudiantes.

\section{Conclusiones:}

Se pudo comprobar que los efectos generados como resultado de la implementación del Proyecto Geniales TIC en el fortalecimiento de las competencias blandas (intrapersonales e interpersonales) es positivo en los directivos y docentes de INETASÍN. Se puede verificar también los cambios efectivos en la Institución Educativa, en su Proyecto Educativo Institucional, PEI, en la labor pedagógica, en el mejor clima organizacional y en el aprendizaje de los estudiantes. La utilización de las TIC para la realización de algunas actividades le agrega más novedad, interés y efectividad al Programa.

Se aplica en el Proyecto lo que afirma Araújo (2017) que:

Quien utiliza su talento predominante para ganarse la vida, desarrolla su inteligencia emocional, perfecciona sus facultades mentales e incorpora el ejercicio de hábitos de la prudencia, justicia, fortaleza y templanza a su manera de ser, se convierte en una persona líder en su área de acción y desarrolla una vida saludable, próspera y feliz ( p. 29).

De esta manera, se reafirma la premisa de GENIALES TIC que expresa que la riqueza y complejidad de cada niño amerita un cambio radical en el enfoque educativo que esté mucho más atento a las características propias de cada cual, a sus distintas combinaciones de diferentes tipos de inteligencias y a todos sus talentos naturales, que fortalezca la inteligencia emocional, el carácter y el proyecto de vida y que este proceso es necesario iniciarlo con los docentes y directivos docentes para que ellos lo realicen con los estudiantes y padres de familia y se realice una verdadera transformación educativa. 
Rev. SINAPSIS, Edición № 14, Vol. 1, Julio 2019

ISSN $1390-9770$

\section{Bibliografía}

Araújo, A. (2017). Aprender a Vivir Mejor (cuarta edición). Cartagena, Colombia.: Imprima.

Arnal, J., Del Rincon, D., \& Latorre, A. (1992). Investigación Educativa. Fundamentos y metodología. Barcelona: Labor.

Buckingham, M., \& Clifton, D. (2011). Ahora, descubra sus fortalezas (Nomos Impresores). Bogotá, Colombia.

Covey, S. R. (2010). Los 7 hábitos de la gente altamente efectiva (Paidós).

Dolev, N., Leshem, S. (2016). Developing emotional intelligence competence among teachers

Recuperado de: http://www.tandfonline.com/doi/full/10.1080/13664530.2016.1207093

Goleman, Daniel. (2013). La Inteligencia Emocional (25a. Edición). Mexico: Nomos impresores.

Guskey, T. (2002). “Does It Make a Difference? Evaluating Professional Development”. Recuperado de https://uknowledge.uky.edu/cgi/viewcontent.cgi?article=1005\&context=edp_facpub

Heckman, J., Kautz, T., (2012) Hard evidence on soft skills https://www.sciencedirect.com/science/article/abs/pii/S0927537112000577?via\%3Dihub

López, P. L. (2004). Población Muestra y Muestreo. Punto Cero, 09(08), 69-74.

Mackey, J., \& Sisodia, R. (2016). Capitalismo consciente (1a.). Barcelona: Editorial Urano.

Navarro, R. (2016). Personas felices y muy efectivas (Instituto Latinoamericano de Liderazgo). Colombia.

OECD. (2016). Education in Colombia. https://doi.org/10.1787/9789264250604-en

Papalia, D., Wendkos, S., Duskin, R.,. (2010). Desarrollo Humano (undécima edición). Recuperado de https://iessb.files.wordpress.com/2015/03/175696292-desarrollo-humano-papalia.pdf

Robinson, K., \& Aronica, L. (2016). El Elemento. Bogotá. 
Rev. SINAPSIS, Edición № 14, Vol. 1, Julio 2019

Robinson, K., \& Aronica, L. (2016). Escuelas Creativas, La Revolución que está transformando la educación. Bogotá: Penguim Random House, Grupo Editorial,.

UNESCO. (2013). Situación Educativa de América Latina y el Caribe: Hacia la educación de calidad para todos al 2015. Recuperado de http://www.unesco.org/new/fileadmin/MULTIMEDIA/FIELD/Santiago/images/SITIEDespanol.pdf 